\title{
La extrusión del dacron subcutáneo como tratamiento de la infección crónica del orificio de salida del catéter de diálisis peritoneal
}

\author{
A. Concepción Gómez Castilla - Ma del Castillo Páez Antunez - Ma Ángeles 0jeda Guerrero - Nuria Aresté Fonsalba \\ - Miguel Ángel Ramírez López - Dolores Fernández Gordillo
}

Enfermero/as

Hospital Universitario Virgen Macarena. Sevilla

\section{Introducción}

Las infecciones del orificio de salida del catéter de diálisis peritoneal, es la morbilidad mas frecuente en este en este tipo de pacientes.

Estudios publicados hablan de un elevado índice de infecciones del orificio/túnel (IOT) que en ocasiones lleva a perdidas del catéter y/o peritonitis relacionadas que pueden ocasionar el deterioro de la membrana peritoneal y la perdida de capacidad dialítica ${ }^{1}$.

Nosotros habitualmente procedemos a la extrusión del dacron externo (DE) o dacron subcutáneo del catéter peritoneal en las infecciones crónicas del orificio/ túnel, (definida por Twardowski como la infección del orificio cuando dura mas de 4 semanas con tratamiento), cuando son rebeldes a los tratamientos con antibiótico en terapia sistémica establecidas mediante antibiograma previo $0^{2,3}$.

La extrusión consiste en extraer quirúrgicamente el tope de dacron externo, respetando el catéter en su posición intraperitoneal, considerando como ventajas el hecho de que el paciente no tenga que interrumpir

\footnotetext{
Dirección para la correspondencia: Concepción Gómez Castilla. C/ Cañada de la viña $n^{\circ} 6$. 41927 Mairena del Aljarafe. Sevilla e-mail atiace@hotmail.com
}

el tratamiento con diálisis peritoneal y evitar al paciente la programación de cirugía mayor que supone el recambio del catéter.

Método de extrusión: Tras anestesia local realizamos una incisión de 2 ó $3 \mathrm{~cm}$. desde el orificio de implantación del catéter y se procede a desbridar los tejidos adheridos al dacron con un Halster mosquito baby recto sin dientes y con ayuda de un bisturí cuidadosamente para no cortar el catéter.

Una vez liberado el dacron, cerramos la incisión mediante puntos de seda en bloque.

En la cura utilizamos fijador de catéter para evitar retroceso del dacron hasta la cicatrización.

En nuestra unidad la hacemos de manera ambulatoria, en la sala de cura de la unidad que utilizamos como quirófano de cirugía local.

Esta técnica la efectúa el medico de la unidad de diálisis peritoneal en la misma unidad y de manera ambulatoria; estableciéndose luego pauta de curas a las 24 y 48 horas para continuar luego pauta personalizada dependiendo de la evolución y las características del paciente y la familia, continuando tratamiento antibiótico con fármaco de elección sensible en antibiograma, al menos durante al primera semana.

En otras ocasiones el dacron extrusa de manera espontánea en pacientes con infección crónica sin que podamos proceder a la extrusión quirúrgica. 
Los casos que presentamos no están relacionados con problemas de fuga y si en algún caso con cambios importante en el índice de masa corporal.

Los catéteres en nuestro servicio son implantados por el cirujano general mediante técnica quirúrgica en línea paramedial y túnel subcutáneo con orificio de salida caudal, ajustados y sin puntos y habitualmente son catéteres de 2 cuff (dacron) con cuello de cisne y porción intraperitoneal recta o en espiral.

\section{Objetivos}

Conocer la supervivencia del catéter de diálisis peritoneal tras la extracción del tope de dacron subcutáneo.

Conocer la incidencia de curación tras la extrusión del dacron externo en infecciones crónicas del orificio de implantación del catéter peritoneal.

Conocer las posibles diferencias entre la extrusión espontánea y la extracción quirúrgica.

\section{Metodología}

Realizamos estudio descriptivo observacional retrospectivo de las extrusiones de dacron subcutáneo habidas en nuestros servicio entre enero de 2004 y diciembre de 2010, que se produjeron en los pacientes portadores de catéter peritoneal, con infección crónica del orificio/ túnel subcutáneo.

Los datos fueron recogidos de la historia del paciente y los registros enfermeros informatizados de nuestra unidad.

Las variables estudiadas fueron: Edad, tipo de diálisis, extrusión de dacron (quirúrgica o espontánea), tiempo de evolución de la IOT hasta la extrusión, germen responsable de la infección del orificio, existencia de peritonitis relacionada (peritonitis detectada durante el periodo de IOT por el mismo germen que ocasionó la infección del orificio), evolución de la peritonitis, control de portadores nasales de estafilococo áureo, evolución de la infección del orificio/túnel tras la extrusión del dacron, antibiótico utilizado y tiempo de tratamiento, motivo de salida de programa y tiempo desde la extrusión.

El tratamiento estadístico se efectuó mediante media y desviación para variable cuantitativa y frecuencias y porcentajes para variables cualitativas. No se efectuó comparación de medias por ser la muestra pequeña debido a que el procedimiento que nos ocupa solo se produce en complicaciones poco frecuentes de la diálisis peritoneal.

\section{Resultados}

Se han revisado 12 pacientes con dacron subcutáneo extrusado. 8 con extrusión quirúrgica y 4 con extrusión espontánea, con una edad media de 56,16 $\pm 15,11$ años, de los que 5 eran mujeres y 7 hombres. En relación al tipo de diálisis 11 pacientes estaban en DPA y sólo 1 paciente en DPAC.

Merece mención aparte, el caso de una mujer de 56 años (no contemplado en este estudio) que durante la extrusión del dacron se cortó el catéter accidentalmente y hubo que recambiarlo en quirófano.

El gráfico 1 refleja el motivo de retirada salida del programa que coincide con el motivo de retirada del catéter, con excepción de una paciente que sufrió una reinfección a los 18meses y hubo que recambiar el catéter que consta entre los fallecidos reinfección

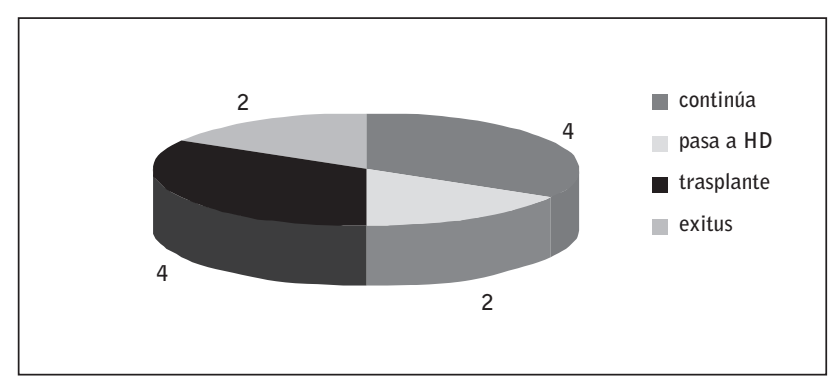

Gráfico 1. Motivo de salida y retirada del catéter

El tiempo medio de seguimiento del catéter fue de $22,25 \pm 22,13$ meses con un rango entre 0 y 57 meses para las extrusiones quirúrgicas y 19,5 $\pm 19,6$ meses con un rango entre 3 y 48 meses para las extrusiones espontáneas. 
El gráfico 2 presenta la distribución de pacientes portadores nasales de estafilococo áureo que en nuestro caso supone el $75 \%$ de los pacientes implicados en el estudio

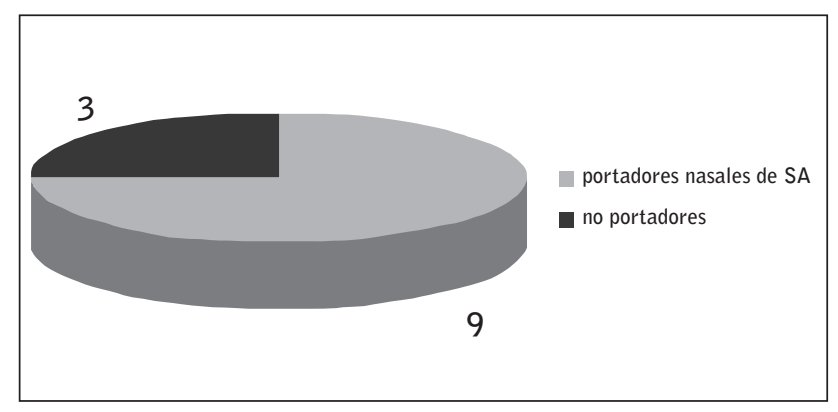

Gráfico 2. Relación de pacientes portadores nasales de estafilococo aureo

La tabla 1 presenta los gérmenes que ocasionaron la IOT y como evolucionaron tras la extrusión de dacron, Destaca la alta implicación del estafilococo áureo en las infecciones crónicas y el hecho de que todos los dacron extrusados quirúrgicamente supusiera la curación de la IOT. Uno de los pacientes tuvo una reinfección a los 18 meses y hubo que recambiar el catéter.

El tiempo de evolución de la IOT hasta la extrusión estuvo en 5,66 $\pm 2,99$ meses pero varió según el mecanismo de extrusión siendo mayor en los dacron de extrusión quirúrgica.
En las extrusiones espontáneas 2 pacientes (50\%) tuvieron mala evolución del orificio de salida aún con la extrusión del dacron, uno se le recambió el catéter y continua en programa y el paciente del corynebacteriun pasó a HD con infección en el orificio por organismo mixto al aparecer una peritonitis por candida que no se consideró relacionada.

En relación al tratamiento utilizado tras la extrusión del dacron se siguió estrictamente las indicaciones del antibiograma y el tiempo medio de tratamiento fue de 26 días con un rango entre 7 y 60 días.

La tabla 2 presenta los gérmenes que ocasionaron las peritonitis relacionadas con las IOT y su evolución, destacando que todas las peritonitis relacionadas con las IOT fueron por estafilococo áureo y merece mención especial la peritonitis que no curó a pesar de que la extrusión del dacron si solucionó la IOT. Esta peritonitis se produce en una mujer de 62 que pasó a hemodiálisis tras un intento de poner un nuevo catéter y observarse por laparoscopia tabicamiento por adherencias.

\section{Discusión}

En nuestro estudio todas las extrusiones espontáneas de dacron se produjeron tras una infección crónica del orificio/túnel.

\begin{tabular}{|l|c|c|c|}
\hline Tipo de extrusión & Germen implicado en la IOT & $\begin{array}{c}\text { Evolución del orificio tras la } \\
\text { extrusión }\end{array}$ & $\begin{array}{c}\text { Tiempo de evolución de la IOT } \\
\text { hasta la extrusión }\end{array}$ \\
\hline E. Quirúrgica N8 & $\begin{array}{c}\text { Estaf. Áureo 5 (62,5\%) } \\
\text { Pseudomonas 3 (37,5\%) }\end{array}$ & $\begin{array}{l}100 \% \text { curación } \\
6,75 \pm 3,10 \text { meses } \\
\text { Rango entre 3 y } 12\end{array}$ \\
\hline E. Espontánea N4 & $\begin{array}{c}\text { Estaf. Áureo 3 (75\%) } \\
\text { Corynebacterium 1 (20\%) }\end{array}$ & $\begin{array}{c}2(50 \%) \text { curación } \\
2(50 \%) \text { no curación }\end{array}$ & $\begin{array}{c}3,5 \pm 1,0 \text { meses } \\
\text { Rango entre 2 y } 4\end{array}$ \\
\hline
\end{tabular}

Tabla 1: Evolución del orificio relacionado con el germen y el tipo de extrusión

\begin{tabular}{|l|c|c|c|}
\hline Tipo de extrusión & $\begin{array}{c}\text { Germen implicado en la } \\
\text { Peritonitis }\end{array}$ & $\begin{array}{c}\text { Peritonitis relacionada con } \\
\text { IOT }\end{array}$ & $\begin{array}{c}\text { Curación de la peritonitis } \\
\text { E. Quirúrgica }\end{array}$ \\
\hline Estaf. Áureo (100\%) & $3(100 \%)$ & $\begin{array}{c}\text { Si } 2(66,6 \%) \\
\text { No } 1(33,3 \%)\end{array}$ \\
\hline E. Espontánea & Estaf. Áureo (100\%) & $1(100 \%)$ & Si $1(100 \%)$ \\
\hline
\end{tabular}

Tabla 2: Peritonitis relacionada con IOT y evolución 
Las extrusiones quirúrgicas siempre se efectuaron en infecciones crónicas del orificio/túnel tras un periodo de tratamiento mínimo de 3 meses y los gérmenes implicados fueron mayoritariamente el estafilococo áureo o la pseudomona.

Cuando se produjo peritonitis relacionada con la infección del orificio fue siempre en pacientes portadores nasales de estafilococo áureo y la peritonitis fue ocasionada por este germen. En un caso no curó y el paciente se transfirió a hemodiálisis tras la retirada del catéter, ya que se observaron múltiples adherencias mediante laparoscopia durante el acto quirúrgico.

En relación a los portadores nasales de estafilococo áureo que en nuestro estudio supone el $75 \%$ de los pacientes de estudio, consideramos necesario insistir en el cumplimiento del protocolo de descontaminación instaurado en la unidad por el alto índice que se produce en la morbilidad infecciosa de estos pacientes ${ }^{4}$.

En nuestros pacientes podemos considerar muy positivo la extrusión del dacron que resolvió el problema de la infección del orificio en le $100 \%$ de los casos, si bien en el $37,5 \%$ de los casos se produjo una peritonitis previa a la extrusión del dacron, por lo que consideramos que quizás deberíamos adelantar el procedimiento para evitar esta posible complicación.

\section{Bibliografía}

1. Rietmann A, Casal MC. Guía de Práctica clínica de Diálisis Peritoneal de la EDTNA/ERCA. Luzern: EDTNA/ERCA; 2009.
2. Muñoz Poyato J. Manual de protocolos y procedimientos de actuación de enfermería nefrológica SEDEN. Madrid: Entheos;2001.

3. Coronel F, Montenegro J, Selga R, Celadilla 0, Tejuca M (Eds). Manual Práctico de Diálisis Peritoneal (SEDEN - SEN). Badalona: Editorial Atrium; 2005.

4. Arrieta J, Bajo MA, Caravaca F, Coronel F, García Pérez H, González Parra E y cols. Guías de práctica clínica en Diálisis Peritoneal. Nefrología 2006;26 (Supl 4).

5. Gómez Castilla AC, Martín Espejo JL, Trujillo Campos C, Laguillo de Castro A, Bejuca Marenco M, López Bermúdez E y cols. Comportamiento del orificio de implantación del catéter en pacientes en diálisis peritoneal en relación a los cuidados. Rev Soc Esp Enferm Nefrol 2007; 10 (4): 263-269.

6. Twardowski ZJ, Prowant BF. Classification of normal and diseased exit-sites. Perit Dial Int 1996;16 Suppl 3:S32-S50.

7. Lima Comas $C$, Alcantud García MJ, Gámez Ráez N, Pastor Palenzuela A, Gómez Marqués G, Morey Molina A. Los apósitos oclusivos en la ducha diaria ¿son capaces de reducir las infecciones del orificio de salida del catéter peritoneal? Rev Soc Esp Enferm Nefrol 2007; 10 (2): 144-148. 